\title{
On the radicals of linear algebraic monoids
}

\author{
Jun Li $\mathbf{~}^{1}$
}

Received: 1 September 2016 / Accepted: 18 November 2016 / Published online: 12 December 2016 (C) The Author(s) 2016. This article is published with open access at Springerlink.com

\begin{abstract}
In this paper we consider the Schwarz radical of linear algebraic semigroups as defined in semigroup theory. We give some new characterizations of the complete regularity, regularity and solvability of irreducible linear algebraic monoids in terms of $S c h w a r z$ radical data. Moreover, we give a generalization about the results of the kernel to the results of completely regular $\mathscr{J}$-classes.
\end{abstract}

Keywords Irreducible algebraic monoid · Kernel · Nilpotent · Radical · Regular • Solvable

\section{Introduction}

Throughout this paper, $\mathbb{Z}^{+}$will denote the set of all positive integers. Let $S$ be a semigroup and $I \subseteq S$ a (two-sided) ideal of $S$. Let $\sqrt{I}$ denote the set of all elements of $S$ which satisfy that some power of them belongs to $I$, i.e.,

$$
\sqrt{I}=\left\{a \in S \mid a^{i} \in I \text { for some } i \in \mathbb{Z}^{+}\right\} .
$$

There are five concepts of radical of $S$ with respect to $I$, called the Cliff ord radical,

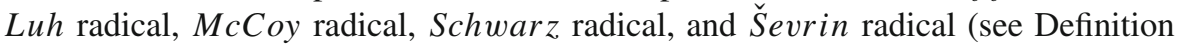
3.1), which are natural extensions of the concepts of radical of a ring. Denote the

\section{Communicated by Jan Okniński.}

$凶$ Jun Li

jimlee509@yahoo.com

1 School of Mathematics and Information Sciences, Guangzhou University, Guangzhou 510006, People's Republic of China 
Cliff ord radical, Luh radical, McCoy radical, Schwarz radical, and $\check{S}$ evrin radical of $S$ with respect to $I$ by $\mathcal{R}^{*}(I), \mathcal{C}(I), \mathcal{M}(I), \mathcal{R}(I), \mathcal{L}(I)$ respectively. If a semigroup $S$ has a $\operatorname{kernel} \operatorname{ker}(S)$ (the minimal ideal of $S$ ), then the $C l i f f$ ord radical, Luh radical, $M c C o y$ radical, Schwarz radical, and $\breve{S}$ evrin radical of $S$ with respect to $\operatorname{ker}(S)$ are simply called the Cliff ord radical, Luh radical, McCoy radical, Schwarz radical, and $\breve{S}$ evrin radical of $S$. Bosăk [3] gives an example in abstract semigroups that all of the radicals mentioned above are distinct from one anther, and shows that for any semigroup $S$ and any ideal $I$ of $S$,

$$
I \subseteq \mathcal{R}(I) \subseteq \mathcal{M}(I) \subseteq \mathcal{L}(I) \subseteq \mathcal{R}^{*}(I) \subseteq \sqrt{I} \subseteq \mathcal{C}(I) \subseteq S
$$

Kme $\check{t}$ [14] prove that $\mathcal{R}^{*}(I)=\sqrt{I}=\mathcal{C}(I)$ if and only if $\sqrt{I}$ is an ideal of $S$. And $S$ is a semilattice of archimedean semigroups (i.e., $b \in S^{1} a S^{1} \Rightarrow b^{i} \in S^{1} a^{2} S^{1}$ for some $i \in \mathbb{Z}^{+}$, for all $a, b \in S$ ), if and only if for any ideal $I$ of $S, \sqrt{I}$ is an ideal of $S$ (see [5] and [15]).

Semigroups which satisfy that the set $\sqrt{I}$ is a semigroup for any ideal $I$, are characterized in [1] by Bogdanović and Ćirić.

In this paper, we consider the above radicals in linear algebraic semigroups. A linear (or affine) algebraic semigroup $S$ over an algebraically closed field $K$ is both an affine variety over $K$ and a semigroup for which the product map $S \times S \rightarrow S$ is a morphism of varieties. Then $S$ has a $\operatorname{kernel} \operatorname{ker}(S)$ (see [22, Theorem 3.28]). Moreover, by [7, II 2.3.3] $S$ is isomorphic to a (Zariski) closed subsemigroup of total $n$ by $n$ matrix monoid $M_{n}(K)$ for some $n \in \mathbb{Z}^{+}$, and is strongly $\pi$-regular (i.e., some power of each element of $S$ lies in a subgroup of $S$ ) by a theorem of Clark (see [22, Theorem 3.18]). Then we have that the Cliff ord radical, McCoy radical, Schwarz radical, and $\breve{S}$ evrin radical of $S$ are coincide, i.e.,

$$
\mathcal{R}(\operatorname{ker}(S))=\mathcal{M}(\operatorname{ker}(S))=\mathcal{L}(\operatorname{ker}(S))=\mathcal{R}^{*}(\operatorname{ker}(S))
$$

Hence we only need to consider the Schwarz radical of a linear algebraic semigroup. In particular, the radical-like property, introduced by J. Luh (see [17]), holds for the Schwarz (or Clifford) radical of a linear algebraic semigroup $S$, that is, the Rees factor semigroup $S / \mathcal{R}(\operatorname{ker}(S))$ of $S$ modulo $\mathcal{R}(\operatorname{ker}(S))$ has zero $S c h w a r z$ (or Clifford) radical.

The following theorems play very important role to study the theory of linear algebraic monoids. Note that if a linear algebraic semigroup $S$ has a zero element, the $S c h w a r z$ radical $\mathcal{R}(\operatorname{ker}(S))$ of $S$ is the maximum nilpotent ideal of $S$ and $\sqrt{\operatorname{ker}(S)}$ is the set of all nilpotent elements of $S$.

Theorem 1.1 [21, Theorem 2.1] Let $S$ be an irreducible linear algebraic semigroup with zero 0 . Then the following conditions are equivalent:

(i) $S$ is completely regular;

(ii) $S$ has no non-zero nilpotent elements (i.e., $\sqrt{\operatorname{ker}(S)}=\{0\}$ );

(iii) $S$ is a monoid and the unit group of $S$ is a torus.

Theorem 1.2 [22, Theorem 7.3] Let $M$ be an irreducible linear algebraic monoid with zero 0 and unit group $G$. Then the following conditions are equivalent: 
(i) $G$ is reductive;

(ii) $M$ is regular;

(iii) $M$ has no non-zero nilpotent ideals (i.e., $\mathcal{R}(\operatorname{ker}(M))=\{0\})$.

Theorem 1.3 [19, Theorem 23] Let $M$ be an irreducible linear algebraic monoid with zero 0 and unit group $G$. Then the following conditions are equivalent:

(i) $G$ is solvable;

(ii) the nilpotents of $M$ form an ideal of $M$ (i.e., $\mathcal{R}(\operatorname{ker}(M))=\sqrt{\operatorname{ker}(M)})$;

(iii) $J^{2} \subseteq J$ for all $J \in \mathcal{U}(M)$.

These facts imply that the structure of irreducible linear algebraic monoids with zero can be characterized in terms of Schwar z radical (or nilpotency) data. In general, for an irreducible linear algebraic monoid $M$, it need not have a zero element, that is, its $\operatorname{kernel} \operatorname{ker}(M)$ is nontrivial. Brion shows in [4, Corollary 3.1.5] that for any irreducible non-affine algebraic monoid (that is, it is non-affine as a variety), its kernel must be nontrivial. The kernel of the linear algebraic monoid $M$ carries a lot of structural information about $M$ as well as of the unit group $G$ which are well studied by Huang (cf. $[9,10,12])$.

The purpose of this paper is to study the structure of linear algebraic monoids in terms of Schwar z radical data. We give generalizations of the above results (Theorems $1.1,1.2,1.3$ ) for an irreducible linear algebraic monoid (without zero). Namely, we prove the following theorem.

Theorem 1.4 Let $M$ be an irreducible linear algebraic monoid with unit group $G$. Then

(i) (Theorem 4.1) $M$ is completely regular if and only if $\sqrt{\operatorname{ker}(M)}=\operatorname{ker}(M)$;

(ii) (Theorem 4.2) $M$ is regular if and only if the Schwarz radical of $M$ is a completely simple semigroup (i.e., $\mathcal{R}(\operatorname{ker}(M))=\operatorname{ker}(M))$;

(iii) (Corollary 4.4) $G$ is solvable if and only if $\sqrt{\operatorname{ker}(M)}$ forms an ideal of $M$ (i.e., $\mathcal{R}(\operatorname{ker}(M))=\sqrt{\operatorname{ker}(M)})$ and a maximal subgroup of $\operatorname{ker}(M)$ is solvable.

Moreover, for any completely regular $\mathscr{J}$-class $J \in \mathcal{U}(M)$, we construct a submonoid $M_{J}$ of $M$ with kernel $J$, defined by

$$
M_{J}=\{a \in M \mid a J \subseteq J\} .
$$

Then $M_{J}$ is a linear algebraic monoid with kernel $J$. Moreover, the unit group of $M_{J}$ is just the unit group of $M$. Hence we can generalize the known results about the kernel of a linear algebraic monoid (see $[9,10,12])$. For instance,

Theorem 1.5 (Corollary 5.5) Let $M$ be an irreducible linear algebraic monoid with unit group $G$. Let $J \in \mathcal{U}(M)$ be completely regular, and $e \in E(J)$. Then

(i) $\operatorname{dim} R(G)=\operatorname{dim} E(J)+\operatorname{dim} R\left(G_{e}\right)+\operatorname{dim} R(e G e)$;

(ii) $\operatorname{dim} R_{u}(G)=\operatorname{dim} E(J)+\operatorname{dim} R_{u}\left(G_{e}\right)+\operatorname{dim} R_{u}(e G e)$;

(iii) $G$ is reductive if and only if $G_{e}$ and $J$ are both reductive groups. 
The article is organized as follows. Section 2 is for notions and notations. In Sect. 3 , we work with various properties of the Schwarz radical of algebraic semigroups. In Sect. 4, we give characterizations of the completely regularity, regularity and solvability of irreducible linear algebraic monoids in terms of Schwarz radical data. In Sect. 5, we generalize the results of Sect. 4 to the case in terms of completely regular $\mathscr{J}$-classes of irreducible linear algebraic monoids.

\section{Preliminaries}

We now assemble some notions and notations. $\mathbb{Z}^{+}$will denote the set of all positive integers. If $X$ is a set, then $|X|$ denotes the cardinality of $X$. Let $S$ be a semigroup. Let $S^{1}:=S \cup\{1\}$ be the natural monoid extension of $S$. The semigroup $S$ is strongly $\pi$-regular (s $\pi \mathrm{r}$ ) if for each $a \in S$, there exists $i \in \mathbb{Z}^{+}$such that $a^{i}$ lies in a subgroup of $S$. If $a, b \in S$, then $a \mid b$ (a divides b) if $x a y=b$ for some $x, y \in S^{1} . S$ is archimedean if for all $a, b \in S, a \mid b^{i}$ for some $i \in \mathbb{Z}^{+}$. Let $S_{\alpha}(\alpha \in \Omega)$ denote a partition of $S$ into subsemigroups. Then $S$ is a semilattice (union) of $S_{\alpha}(\alpha \in \Omega)$ if for all $\alpha, \beta \in \Omega$, there exists $\gamma \in \Omega$ such that $S_{\alpha} S_{\beta} \cup S_{\beta} S_{\alpha} \subseteq S_{\gamma}$. According to [22, Theorem 1.15], S is a semilattice of archimedean semigroups if and only if for all $a, b \in S, a \mid b$ implies $a^{2} \mid b^{i}$ for some $i \in \mathbb{Z}^{+}$. Let $E(S)$ denote the set of all idempotents of $S$. Let $e \in E(S)$. We denote by $J_{e}, L_{e}, R_{e}$ and $H_{e}$ the $\mathcal{J}$-, $\mathcal{L}$-, $\mathcal{R}$ - and $\mathcal{H}$-classes of $e$ in $S$ under Green's relations, respectively (see [22, Chap. 1]). Suppose $S$ is an s $\pi$ r-semigroup and $J$ is a $\mathcal{J}$-class of $S$. Then $J$ is regular if $E(J) \neq \varnothing$. Moreover, $J$ is completely regular if $J$ is regular and $J^{2} \subseteq J$. Let $\mathcal{U}(S)$ be the set of all regular $\mathcal{J}$-classes of $S$. For any $J_{1}, J_{2} \in \mathcal{U}(S)$, we denote $J_{1} \leq J_{2}$ if $a_{2} \mid a_{1}$ for some (all) $a_{i} \in J_{i}, i=1,2$. We write $\mathcal{U}(S)$ for the partially ordered set $(\mathcal{U}(S), \leq)$. Let $\varnothing \neq I \subseteq S$. Then $I$ is a right ideal of $S$ if $I S \subseteq I$; $I$ is a left ideal of $S$ if $S I \subseteq I$; $I$ is an ideal of $S$ if $S^{1} I S^{1} \subseteq I$. The minimum ideal of $S$, if it exists, is called the kernel of $S$, denoted by $\operatorname{ker}(S)$. A completely simple semigroup $S$ is an s $\pi$ r-semigroup with no ideals other than $S$.

Let $K$ denote a fixed algebraically closed field. $M_{n}(K)$ will denote the algebra of all $n \times n$ matrices over $K$, and $G L_{n}(K)$ its unit group. Let $S$ be a Zariski closed subsemigroup of $M_{n}(K)$. If $e \in E(S)$ and $a \in S$, then we let $\operatorname{det}_{e}(a)=\operatorname{det}($ eae + $1-e)$. $\operatorname{Thus}_{\operatorname{det}}(a) \neq 0$ if and only if eae $\in H_{e}$ by [22, Remark 3.23].

Let $M$ be an irreducible linear algebraic monoid over $K$ with unit group $G . M$ is regular (resp. completely regular) if it is so as a semigroup. We call $M$ reductive (resp. semisimple, solvable, nilpotent, a d-monoid) if its unit group is reductive (resp. reductive with center 1-dimensional, solvable, nilpotent, a torus). We write $R(G)$ (resp. $R_{u}(G)$ ) for the radical (resp. unipotent radical) of $G$. The $\operatorname{rank}$ of $G$, denoted $\operatorname{rank}(G)$, is referred to as the dimension of a maximal torus of $G$. Let $W(G)$ denote the weyl group of $G$. Then by [13, Proposition 24.1A, Corollary 25.2C], $W(G)$ is finite, and $G$ is solvable if and only if $|W(G)|=1$. For a subset $V$ of $M$, denote by $\bar{V}$ the Zariski closure of $V$ in $M$. If $N$ is a closed algebraic subsemigroup of $M$, let $N^{c}$ be the identity component of $N$. If $e \in E(M)$, then we denote $M_{e}=\{a \in M \mid e a=a e=e\}^{c}$, $G^{r}(e)=\{x \in G \mid x e=e\}, G^{l}(e)=\{x \in G \mid e x=e\}$, and $G_{e}=\left(G^{r}(e) \cap G^{l}(e)\right)^{c}$. For any subset $X$ of $M, C_{X}^{r}(e)=\{a \in X \mid a e=e a e\}, C_{X}^{l}(e)=\{a \in X \mid e a=e a e\}$, $C_{X}(e)=\{a \in X \mid a e=e a\}$. Let $T$ be a maximal torus of $G$. Then $\Lambda \subseteq E(\bar{T})$ is 
a cross-section lattice of $M$, if $|\Lambda \cap J|=1$ for all $J \in \mathcal{U}(M)$ and $J_{e} \geq J_{f}$ implies $e \geq f$ for all $e, f \in \Lambda$. If $J \in \mathcal{U}(M)$, then the width of $J, \omega(J)=|J \cap E(\bar{T})|$. If $e \in E(J), \omega(e)=\omega(J)$. For completely regular $\mathcal{J}$-classes, we have the following characterizations.

Theorem 2.1 [22, Remark 1.7(iii)] Let $S$ be a linear algebraic semigroup, $J \in \mathcal{U}(S)$. Then the following conditions are equivalent:

(i) $J$ is completely regular;

(ii) $J$ is completely simple;

(iii) $J^{2} \subseteq J$;

(iv) $E(J)^{2} \subseteq J$.

Theorem 2.2 [22, Theorem 6.30, Corollary 6.34] Let $M$ be an irreducible linear algebraic monoid with unit group $G, J \in \mathcal{U}(M)$ and $e \in E(J)$. Then the following conditions are equivalent:

(i) $J$ is completely regular;

(ii) $E(J) \subseteq \bar{B}$ for some Borel subgroup $B$ of $G$;

(iii) $\omega(e)=1$;

(iv) $e \in E(\overline{R(G)})$;

(v) $G=C_{G}^{l}(e) C_{G}^{r}(e)$;

(vi) $e G e$ is the $\mathcal{H}$-class of $e$.

$[22,23]$ are our primary references for algebraic monoid theory, and $[2,13,26]$ for algebraic group theory.

\section{The radicals of linear algebraic semigroups}

Let $S$ be a linear algebraic semigroup and $I$ a (two-sided) ideal of $S$. Let $\operatorname{ker}(S)$ be the kernel of $S$ (the minimum ideal of $S$ ). The following concepts are used for abstract semigroups which are defined analogously to these concepts for rings.

An element $a$ of $S$ is termed a nilpotent element of $S$ with respect to $I$ if $a^{i} \in I$ for some $i \in \mathbb{Z}^{+}$. Let $\sqrt{I}$ denote the set of all nilpotent elements of $S$ with respect to $I$, i.e.,

$$
\sqrt{I}=\left\{a \in S \mid a^{i} \in I \text { for some } i \in \mathbb{Z}^{+}\right\} .
$$

By [22, Corollary 3.30], the set $\sqrt{I}$ is closed in $S$. An ideal (left or right), or a subsemigroup $A$ of $S$ is nilpotent with respect to $I$ if $A^{i} \subseteq I$ for some $i \in \mathbb{Z}^{+}$, and is nil with respect to $I$ if every element of $A$ is a nilpotent element of $S$ with respect to $I$. For simplicity, an ideal $A$ (left or right) is nilpotent (resp., nil) if $A$ is nilpotent (resp., nil) with respect to $\operatorname{ker}(S)$. An ideal $A$ of $S$ is locally nilpotent with respect to $I$ if every subsemigroup $S_{1} \subseteq S$, generated by a finite number of elements of $A$, is nilpotent with respect to $I$. An ideal $P$ of $S$ is called a prime ideal of $S$ if $I_{1} I_{2} \subseteq P$ implies that $I_{1} \subseteq P$ or $I_{2} \subseteq P$ where $I_{1}$ and $I_{2}$ are ideals of $S$. An ideal $P$ of $S$ is called a completely prime ideal of $S$ if for any two elements $a, b \in S, a b \in I$ implies 
that $a \in I$ or $b \in I$. Evidently a completely prime ideal is a prime ideal. Now we define some radicals of $S$ with respect to $I$.

Definition 3.1 Let $S$ be a linear algebraic semigroup, and $I$ an ideal of $S$.

(i) The union $\mathcal{L}(I)$ of all locally nilpotent ideals of $S$ with respect to $I$ is called the Sevrin (or locally nilpotent) radical of $S$ with respect to $I$.

(ii) The union $\mathcal{R}^{*}(I)$ of all nil ideals of $S$ with respect to $I$ is called the Clifford (or nil) radical of $S$ with respect to $I$ [6].

(iii) The union $\mathcal{R}(I)$ of all nilpotent ideals of $S$ with respect to $I$ is called the $S c h w a r z$ (or nilpotent) radical of $S$ with respect to $I$ [24].

(iv) The intersection $\mathcal{M}(I)$ of all prime ideals of $S$ containing $I$ is called the McCoy (or prime) radical of $S$ with respect to $I$ [17].

(v) The intersection $\mathcal{C}(I)$ of all completely prime ideals of $S$ containing $I$ is called the $L u h$ (or completely prime) radical of $S$ with respect to $I$ [17].

Obviously, every nilpotent (left or right) ideal of $S$ with respect to $I$ is nil with respect to $I$. By [22, Corollary 3.16], there exists $n \in \mathbb{Z}^{+}$such that $S$ is isomorphic to a (Zariski) closed subsemigroup of $M_{n}(K)$, and thus for all $a \in S, a^{n}$ lies in a subgroup of $S$ following [22, Theorem 3.18]. Hence

$$
\sqrt{I}=\left\{a \in S \mid a^{n} \in I\right\} .
$$

Then every nil (left or right) ideal of $S$ with respect to $I$ is nilpotent with respect to $I$. Therefore, an ideal of $S$ is nil with respect to $I$ if and only if it is nilpotent with respect to $I$. Thus $\mathcal{R}(I)=\mathcal{R}^{*}(I)$. It is known that (see [3])

$$
I \subseteq \mathcal{R}(I) \subseteq \mathcal{M}(I) \subseteq \mathcal{L}(I) \subseteq \mathcal{R}^{*}(I) \subseteq \sqrt{I} \subseteq \mathcal{C}(I) \subseteq S
$$

So the Clifford, McCoy, Sevrin, Schwarz radicals of $S$ with respect to I coincide, that is,

$$
\mathcal{R}(I)=\mathcal{M}(I)=\mathcal{L}(I)=\mathcal{R}^{*}(I)
$$

Hence we only consider the Schwarz radical of a linear algebraic semigroup $S$ with respect to an ideal $I$.

Throughout this paper, we use the notation $\mathcal{R}(I)$ to denote the Schwarz radical of a linear algebraic semigroup $S$ with respect to an ideal $I$ of $S$. We write $\mathcal{R}_{S}(I)$, if we want to specify $S$. For simplicity, the $S c h w a r z$ radical of $S$ with respect to $\operatorname{ker}(S)$ is called the $S c h w a r z$ radical of $S$, denoted by $\mathcal{R} \operatorname{ker}(S)$. Clearly, $\mathcal{R}(I)$ is the largest nilpotent (or nil) ideal of $S$ with respect to $I$. Since every ideal of $S$ contained in $\sqrt{I}$ is nil with respect to $I$, it is easy to see that $\mathcal{R}(I)$ is also the largest ideal of $S$ contained in $\sqrt{I}$. The following lemma shows that $\mathcal{R}(I)$ is the largest nilpotent (or nil) left (or right) ideal of $S$ with respect to $I$.

Lemma 3.2 Let $S$ be a linear algebraic semigroup and I an ideal of $S$. Then $\mathcal{R}(I)$ contains every nilpotent left (or right) ideal of $S$ with respect to $I$. 
Proof Let $A$ be a nilpotent left ideal of $S$ with respect to $I$. Then there exists some $i \in \mathbb{Z}^{+}$such that $A^{i} \subseteq I$. So $\left(A S^{1}\right)^{i} \subseteq A^{i} S^{1} \subseteq I S^{1} \subseteq I$. Hence $A S^{1}$ is a nilpotent ideal of $S$ with respect to $I$. Therefore $A \subseteq A S^{1} \subseteq \mathcal{R}(I)$. Similarly, we have that every nilpotent right ideal of $S$ with respect to $I$ is also contained in $\mathcal{R}(I)$.

By Lemma 3.2, we have the following lemma directly.

Lemma 3.3 Let $S$ be a closed subsemigroup of $M_{n}(K)$, I an ideal of $S$. Then

$$
\begin{aligned}
\mathcal{R}(I) & =\left\{a \in S \mid(x a y)^{n} \in I \text { for all } x, y \in S^{1}\right\} \\
& =\left\{a \in S \mid(a y)^{n} \in I \text { for all } y \in S^{1}\right\} \\
& =\left\{a \in S \mid(x a)^{n} \in I \text { for all } x \in S^{1}\right\}
\end{aligned}
$$

Proposition 3.4 Let $M$ be a linear algebraic monoid with unit group $G$ and let I be an ideal of $M$. Then

$$
\begin{aligned}
\mathcal{R}(I) & =\{a \in M \mid G a G \subseteq \sqrt{I}\} \\
& =\{a \in M \mid a G \subseteq \sqrt{I}\} \\
& =\{a \in M \mid G a \subseteq \sqrt{I}\} .
\end{aligned}
$$

Proof Without loss of generality, we may assume that $M$ is a closed submonoid of $M_{n}(K)$ for some $n \in \mathbb{Z}^{+}$. By Lemma 3.3, we have

$$
\mathcal{R}(I)=\{a \in M \mid M a M \subseteq \sqrt{I}\}=\{a \in M \mid a M \subseteq \sqrt{I}\}=\{a \in M \mid M a \subseteq \sqrt{I}\} .
$$

If $a \in \mathcal{R}(I)$, then $G a G \subseteq M a M \subseteq \sqrt{I}$. If $b \in M$ with $G b G \subseteq \sqrt{I}$, since $\sqrt{I}$ is closed in $M$, we have

$$
M b M \subseteq \overline{G b G}=\overline{M b M} \subseteq \sqrt{I},
$$

which implies $b \in \mathcal{R}(I)$. Therefore,

$$
\mathcal{R}(I)=\{a \in M \mid G a G \subseteq \sqrt{I}\} .
$$

Similarly, we can get

$$
\mathcal{R}(I)=\{a \in M \mid a G \subseteq \sqrt{I}\}=\{a \in M \mid G a \subseteq \sqrt{I}\}
$$

Proposition 3.5 Let $S$ be a linear algebraic semigroup, I an ideal of $S$ and $e \in E(S)$. Then $\mathcal{R}(I)$ is closed in $S$. 
In particular,

$$
\mathcal{R}(\operatorname{SeS})=\bigcap_{f \in E(S) \backslash E(S e S)} I(f),
$$

where $I(f)=\{a \in S \mid a \nmid f\}$.

Proof Consider the product $\mu: S \times S \rightarrow S$ by $\mu(a, b)=a b$ for any $a, b \in S$. As $\mu(S \times \mathcal{R}(I))=S \mathcal{R}(I) \subseteq \mathcal{R}(I)$, we deduce that

$$
\mu(S \times \overline{\mathcal{R}(I)})=\mu(\overline{S \times \mathcal{R}(I)}) \subseteq \overline{\mu(S \times \mathcal{R}(I))} \subseteq \overline{\mathcal{R}(I)}
$$

Similarly, we can get $\mu(\overline{\mathcal{R}(I)} \times S) \subseteq \overline{\mathcal{R}(I)}$. So $S \overline{\mathcal{R}(I)} S \subseteq \overline{\mathcal{R}(I)}$, which implies that $\overline{\mathcal{R}(I)}$ is an ideal of $S$. Since $\sqrt{I}$ is closed in $S, \overline{\mathcal{R}(I)} \subseteq \sqrt{I}$. By the maximality of $\mathcal{R}(I)$, we get $\overline{\mathcal{R}(I)}=\mathcal{R}(I)$, thus $\mathcal{R}(I)$ is closed in $S$.

Let $a \in \mathcal{R}(S e S)$ and $f \in E(S) \backslash E(S e S)$. If $a \mid f$, then $f \in S a S \subseteq \mathcal{R}(\operatorname{SeS})$, thus $f \in E(\mathcal{R}(\operatorname{SeS}))=E(\operatorname{Se} S)$, a contradiction. So $a \nmid f$, and thus $a \in I(f)$. Hence

$$
\mathcal{R}(S e S) \subseteq \bigcap_{f \in E(S) \backslash E(S e S)} I(f) .
$$

On the other hand, suppose $a \in S$ and $a \nmid f$ for every $f \in E(S) \backslash E(S e S)$. We claim that $a \in \mathcal{R}(S e S)$. In fact, if not, there exists $y \in S$, such that $(a y)^{n} \notin S e S$ for some $n \in \mathbb{Z}^{+}$by Lemma 3.3. Thus there exits $f \in E(S) \backslash E(\operatorname{SeS})$ such that $(a y)^{n} \in H_{f}$. Then $a \mid f$, a contradiction. So $a \in \mathcal{R}(\operatorname{SeS})$. Therefore,

$$
\mathcal{R}(\operatorname{SeS})=\bigcap_{f \in E(S) \backslash E(S e S)} I(f) .
$$

For a linear algebraic semigroup $S$ with $\operatorname{kernel} \operatorname{ker}(S)$, recall that an idempotent $e \in$ $E(S)$ is called primitive if $E(e S e) \backslash E(\operatorname{ker}(S))=\{e\}$, that is, for any $f \in E(S)$, $e \gtrless f$ implies $f \in E(\operatorname{ker}(S))$. The following corollary gives a characterization of the $S c h w a r z$ radical of an irreducible linear algebraic monoid in terms of primitive idempotents as follows.

Corollary 3.6 Let $M$ be an irreducible linear algebraic monoid with unit group $G$. Let $T$ be a maximal torus of $G, \Lambda \subseteq E(\bar{T})$ a cross-section lattice of $M$. Then

$$
\mathcal{R} \operatorname{ker}(M)=\bigcap_{e \in \Lambda_{0}} I(e)
$$

where $I(e)=\{a \in M \mid a \nmid e\}$ and $\Lambda_{0}=\{e \in \Lambda \mid e$ is primitive $\}$. 
Proof First, we claim that if $e, f \in E(M)$ with $e \leq f$, then $I(e) \subseteq I(f)$. In fact, for any $a \in I(e)$, if $a \notin I(f)$, then $a \mid f$. Since $f \mid e$, we have $a \mid e$, a contradiction. Let $\Omega=\{e \in E(M) \mid e$ is primitive $\}$. By [22, Corollary 6.9, Theorem 6.20], for any $e \in E(M) \backslash E(\operatorname{ker}(M))$, there exists $e^{\prime} \in \Omega$ such that $e \geq e^{\prime}$. Hence, by Proposition 3.5 ,

$$
\mathcal{R} \operatorname{ker}(M)=\bigcap_{e \in E(M) \backslash E(\operatorname{ker}(M))} I(e)=\bigcap_{e \in \Omega} I(e) .
$$

Next, we claim that if $e, f \in E(M)$ with $J_{e}=J_{f}$, then $I(e)=I(f)$. Since $J_{e}=J_{f}$, by [22, Corollary 6.8], there exists $x \in G$ such that $x^{-1} e x=f$. Let $a \in I(e)$. If $a \mid f$, then $f \in M a M$. Thus $e=x f x^{-1} \in M a M$, which implies $a \mid e$, a contradiction. So $I(e) \subseteq I(f)$. Similarly, we get $I(f) \subseteq I(e)$. Therefore, $I(e)=I(f)$. According to [22, Corollary 6.10], $E(M)=\bigcup_{x \in G} x^{-1} E(\bar{T}) x$. So

$$
\mathcal{R} \operatorname{ker}(M)=\bigcap_{e \in \Omega} I(e)=\bigcap_{e \in \Lambda_{0}} I(e)
$$

where $\Lambda_{0}=\{e \in \Lambda \mid e$ is primitive $\}$.

Remark 3.7 (i) For an irreducible linear algebraic monoid $M$ with unit group $G$, if $e \in E(M)$ such that the $\mathscr{J}$-class $J_{e}$ of $M$ is completely regular, then by the proof of Proposition 5.8,

$$
I(e)=\{a \in M \mid a \nmid e\}=\left\{a \in M \mid \operatorname{det}_{e}(a)=0\right\},
$$

and $I(e)$ is the union of some irreducible components of the non-units of $M$. In particular, if $M$ is solvable with zero, then for any $e \in E(M)$, the $\mathscr{J}$-class $J_{e}$ of $M$ is completely regular by [22, Corollary 6.32]. Hence following Corollary 3.6, we obtain that

$$
\mathcal{R} \operatorname{ker}(M)=\left\{a \in M \mid \operatorname{det}_{e_{i}}(a)=0,1 \leq i \leq n\right\},
$$

Where $\left\{e_{i} \mid 1 \leq i \leq n\right\}$ are the set of all primitive idempotents of $E(\bar{T})$ for a maximal torus $T$ of $G$.

For example, let $M$ be the set of all upper triangular matrices in $M_{3}(K)$, i.e.,

$$
M=\left(\begin{array}{ccc}
K & K & K \\
0 & K & K \\
0 & 0 & K
\end{array}\right):=\left\{\left(\begin{array}{ccc}
a_{1} & b_{1} & c \\
0 & a_{2} & b_{2} \\
0 & 0 & a_{3}
\end{array}\right) \mid a_{i}, b_{j}, c \in K, 1 \leq i \leq 3,1 \leq j \leq 2\right\}
$$


Then $\operatorname{ker}(M)=\{0\}$, and the Schwarz radical of $M, \mathcal{R} \operatorname{ker}(M)=\left(\begin{array}{ccc}0 & K & K \\ 0 & 0 & K \\ 0 & 0 & 0\end{array}\right)$.

Choose

$$
e_{1}=\left(\begin{array}{lll}
1 & 0 & 0 \\
0 & 0 & 0 \\
0 & 0 & 0
\end{array}\right), e_{2}=\left(\begin{array}{lll}
0 & 0 & 0 \\
0 & 1 & 0 \\
0 & 0 & 0
\end{array}\right), e_{3}=\left(\begin{array}{lll}
0 & 0 & 0 \\
0 & 0 & 0 \\
0 & 0 & 1
\end{array}\right)
$$

Thus

$I\left(e_{1}\right)=\left(\begin{array}{lll}0 & K & K \\ 0 & K & K \\ 0 & 0 & K\end{array}\right), I\left(e_{2}\right)=\left(\begin{array}{ccc}K & K & K \\ 0 & 0 & K \\ 0 & 0 & K\end{array}\right), I\left(e_{3}\right)=\left(\begin{array}{ccc}K & K & K \\ 0 & K & K \\ 0 & 0 & 0\end{array}\right)$

So

$$
\mathcal{R} \operatorname{ker}(M)=\bigcap_{1 \leq i \leq 3} I\left(e_{i}\right)
$$

Note that the $e_{i}$ s are primitive idempotents of $M$, and the $I\left(e_{i}\right)$ s are exactly the irreducible components of the set of all non-units of $M$.

(ii) Even thought the Schwarz radical of any linear algebraic semigroup is closed which is showed in Proposition 3.5, it may not be irreducible. Let

$$
M=\left\{a \otimes b \mid a, b \in\left(\begin{array}{cc}
K & K \\
0 & K
\end{array}\right)\right\}, E_{12}=\left(\begin{array}{ll}
0 & 1 \\
0 & 0
\end{array}\right) .
$$

Then the $S c h w a r z$ radical of $M, \mathcal{R} \operatorname{ker}(M)=S_{1} \cup S_{2}$, where

$$
\begin{aligned}
& S_{1}=\left\{a \otimes E_{12} \mid a \in\left(\begin{array}{ll}
K & K \\
0 & K
\end{array}\right)\right\}, \\
& S_{2}=\left\{E_{12} \otimes b \mid b \in\left(\begin{array}{ll}
K & K \\
0 & K
\end{array}\right)\right\} .
\end{aligned}
$$

So $\mathcal{R} \operatorname{ker}(M)$ is reducible.

Now we discuss the properties of the minimal non-nilpotent ideals of linear algebraic semigroups determined by primitive idempotents. We have the following proposition which is similar to the result of [16, Theorem 1] in compact semigroups with zero.

Proposition 3.8 Let $S$ be a linear algebraic semigroup, $e \in E(S)$. Then the following are equivalent:

(i) $e$ is a primitive idempotent of $S$;

(ii) the set e $S e \backslash \sqrt{\operatorname{ker}(S)}$ is a group;

(iii) Se is a minimal non-nilpotent left ideal of $S$; 
(iv) eS is a minimal non-nilpotent right ideal of $S$;

(v) SeS is a minimal non-nilpotent ideal of $S$;

(vi) each idempotent in $S e S \backslash \operatorname{ker}(S)$ is a primitive idempotent of $S$.

Proof (i) $\Longrightarrow$ (ii): Assume that $e$ is a primitive idempotent of $S$. Then we have $H_{e} \subseteq e S e \backslash \sqrt{\operatorname{ker}(S)}$. Let $a \in e S e \backslash \sqrt{\operatorname{ker}(S)}$. Then $a^{m} \in H_{f}$ for some $f \in E(e S e)$ and some $m \in \mathbb{Z}^{+}$. Since $a \notin \sqrt{\operatorname{ker}(S)}$, we have $f \notin E(\operatorname{ker}(S))$, which implies $f=e$. Hence $a^{m} \in H_{e}$, and then $a \in H_{e}$. Therefore, the set $e S e \backslash \sqrt{\operatorname{ker}(S)}=H_{e}$ is the maximal subgroup of $S$ containing $e$.

(ii) $\Longrightarrow$ (iii): Assume (ii) and that $I_{l}$ is a left non-nilpotent ideal of $S$ contained in $S e$. We claim that there exists an idempotent $f \in E\left(I_{l}\right) \backslash E(\operatorname{ker}(S))$. Since $S$ is a linear algebraic semigroup, $I_{l}$ is a non-nil ideal of $S$. Thus there exists $x \in I_{l} \backslash \sqrt{\operatorname{ker}(S)}$. But by [22, Theorem 3.18], there exist $f \in E(S)$ and $m \in \mathbb{Z}^{+}$ such that $x^{m} \in H_{f} \cap I_{l}$. So there exists $y \in S$ such that $y x^{m}=f \in I_{l}$, since $I_{l}$ is a left ideal of $S$. Since $x \notin \sqrt{\operatorname{ker}(S)}$ and $x^{m} \in H_{f}$, we have $f \notin$ $E(\operatorname{ker}(S))$. Now $f \in S e$, implying $f=f e$. Then $e f=e f e f \in E(e S e)$. Since $f e f=f \notin E(\operatorname{ker}(S))$, ef $\notin E(\operatorname{ker}(S))$. Since $e S e \backslash \sqrt{\operatorname{ker}(S)}$ is a group and ef $\in E(e S e \backslash \sqrt{\operatorname{ker}(S)}), e=e f \in I_{l}$. Hence $I_{l}=S e$.

(iii) $\Longrightarrow$ (v): Suppose $I$ is a non-nilpotent ideal of $S$ contained in $S e S$. Then $I$ is a non-nil ideal of $S$, as $S$ is a linear algebraic semigroup. Thus there exist $x \in$ $I \backslash \sqrt{\operatorname{ker}(S)}$ and $m \in \mathbb{Z}^{+}$such that $x^{m} \in H_{f} \cap I$ for some $f \in E(S) \backslash E(\operatorname{ker}(S))$. So there exists some $y \in S$ such that $f=y x^{m} \in I \subseteq S e S$. Hence there exist $a, b \in S$ such that $f=a e b$. Let $g=$ bfae $\in I$. Then $g^{2}=$ bfaebfae $=$ $b f a e=g \in E(S e)$. Since $f \notin E(\operatorname{ker}(S))$ and $f=a e g b, g \notin E(\operatorname{ker}(S))$. By the minimality of $S e$, we have $S e=S g$. Thus $S e S=S g S \subseteq I$, implying $I=\operatorname{SeS}$

$(\mathrm{v}) \Longrightarrow$ (i): Assume (v). If $e$ were not primitive, there would exist $f \in E(e S e) \backslash$ $E(\operatorname{ker}(S))$ such that $e \neq f$. Thus $S f S$ in a non-nilpotent ideal of $S$ contained in $S e S$. By the minimality of $S e S, S f S=S e S$. Hence $f \in e S e \cap J_{e}$. By [22, Theorem 1.4(iii)], $e S e \cap J_{e}=H_{e}$. Then $f=e$, a contradiction. Therefore, $e$ is a primitive idempotent of $S$;

The result (vi) $\Longleftrightarrow$ (i) follows from (v) $\Longleftrightarrow$ (i). And by symmetry, we finish the proof.

In a similar way in [6, Theorem 1.1] and by Proposition 3.8, we have the following proposition.

Proposition 3.9 Let $S$ be a linear algebraic semigroup and let I be a minimal nonnilpotent ideal of $S$. Then any proper ideal of I is nilpotent. In particular, there exists a primitive idempotent $e \in E(S)$ such that $I=S e S$.

Proof Note that the kernel of $I$ is equal to $\operatorname{ker}(S)$ by [25]. Let $A$ be a proper ideal of $I$. Suppose (by way of contradiction) $A$ is not a nilpotent ideal of $I$ with respect to $\operatorname{ker}(S)$. Then $I A I$ is a two-sided ideal of $S$ contained in $I$, and $I A I \subseteq A \subsetneq I$. By the minimality of $I, I A I$ is a nilpotent ideal of $S$, and thus $I A I \subseteq \mathcal{R} \operatorname{ker}(S)$. Then $(I A)^{2}=I A I A \subseteq \mathcal{R} \operatorname{ker}(S) A \subseteq \mathcal{R} \operatorname{ker}(S)$. So $I A$ is a nilpotent left ideal of $S$. Similarly, $A I$ is a nilpotent right ideal of $S$. 
Now, $S A S$ is a two-sided ideal of $S$ contained in $I$. By the minimality of $I$, we have that $S A S$ is a nilpotent ideal of $S$ or $S A S=I$. In either event, since $I A$ is nilpotent, we have that $(S A)^{2}=S A S A$ is nilpotent. Hence $S A$ is a nilpotent left ideal of $S$. Therefore, $S A \subseteq \mathcal{R} \operatorname{ker}(S)$. Thus there exists some $m \in \mathbb{Z}^{+}$such that $(S A)^{m} \subseteq \operatorname{ker}(S)$. So $A^{2 m}=(A \cdot A)^{m} \subseteq(S \cdot A)^{m} \subseteq \operatorname{ker}(S)$. Hence $A$ is a nilpotent ideal of $I$, a contradiction. Therefore, we have that any proper ideal of $I$ is nilpotent.

Since $I$ is non-nilpotent, $I$ is non-nil, and thus there exists $a \in I$ such that $a \notin$ $\sqrt{\operatorname{ker}(S)}$. By [22, Theorem 3.18], there exist some $n \in \mathbb{Z}^{+}$and $e \in E(S)$ such that $a^{n} \in H_{e}$. Clearly, $e \notin E(\operatorname{ker}(S))$. Hence there exists $b \in S$ such that $e=a^{n} b$, which implies $e \in E(I) \backslash E(\operatorname{ker}(S))$. So $\operatorname{Se} S$ is a non-nilpotent ideal of $S$ contained in $I$, which implies $S e S=I$. By Proposition 3.8, $e$ is a primitive idempotent of $S$.

\section{The structure of linear algebraic monoids in terms of Schwarz radical data}

For an irreducible linear monoid $M$, it is easy to see that

$$
\operatorname{ker}(M) \subseteq \mathcal{R} \operatorname{ker}(M) \subseteq \sqrt{\operatorname{ker}(M)}
$$

In the Sect. 4.1, we give a characterization of the condition that $\operatorname{ker}(M)=\mathcal{R} \operatorname{ker}(M)$. And in the Sect. 4.2, we give a characterization of the condition that $\mathcal{R} \operatorname{ker}(M)=$ $\sqrt{\operatorname{ker}(M)}$.

\subsection{Completely regularity and regularity conditions}

Theorem 4.1 Let $M$ be an irreducible linear algebraic monoid. Then the following are equivalent:

(i) $M$ is completely regular;

(ii) $\sqrt{\operatorname{ker}(M)}=\operatorname{ker}(M)$;

(iii) $\left\{a \in M \mid a^{i}=f\right.$ for some $\left.i \in \mathbb{Z}^{+}\right\} \subseteq f G f$ for every $f \in E(\operatorname{ker}(M))$.

Proof (i) $\Longrightarrow$ (ii). Suppose $M$ is completely regular and $a \in \sqrt{\operatorname{ker}(M)}$. Then there exists $f \in E(\operatorname{ker}(M))$ such that $a \in H_{f}$. Hence $a \in \operatorname{ker}(M)$ as $H_{f} \subseteq \operatorname{ker}(M)$. So $\sqrt{\operatorname{ker}(M)}=\operatorname{ker}(M)$.

(ii) $\Longrightarrow$ (iii). Assume that $\sqrt{\operatorname{ker}(M)}=\operatorname{ker}(M)$. Let $f \in E\left(\operatorname{ker}(M)\right.$. If $a^{i}=f$ for some $i \in \mathbb{Z}^{+}$, then $a \in \sqrt{\operatorname{ker}(M)}$, and thus $a \in \operatorname{ker}(M)$. So there exists $e \in E(M)$ such that $a \in H_{e}$. Then $a^{i}=f \in H_{e}$ which implies $e=f$. Therefore, $a \in H_{f}$. By Theorem 2.2, we have that $H_{f}=f G f$. Therefore, $a \in f G f$.

(iii) $\Longrightarrow$ (i). Suppose (iii) holds. For any $f \in E(\operatorname{ker}(M)$, if a is a nilpotent element of $M_{f}$, then $a^{i}=f$ for some $i \in \mathbb{Z}^{+}$. Thus $a \in f G f \cap M_{f}=\{f\}$, then $a=f$. This shows that $M_{f}$ has no non-zero nilpotent elements, hence by [22, Theorem 5.12], $M_{f}$ is completely regular. Following [22, Theorem 7.4], we obtain that $M$ is regular. Moreover, by[18, Theorem 3.7], $E\left(M_{f}\right)=\{e \in E(M) \mid e \geq f\}$ is finite. Therefore, $M$ is completely regular following [20, Theorem 3.10]. 
Theorem 4.2 Let $M$ be an irreducible linear algebraic monoid. Then $M$ is regular if and only if the Schwarz radical of $M$ is completely simple (i.e., $\mathcal{R} \operatorname{ker}(M)=$ $\operatorname{ker}(M))$.

Proof By [22, Theorem 3.15], $M$ is isomorphic to a closed submonoid of $M_{n}(K)$ for some $n \in \mathbb{Z}^{+}$, thus $\sqrt{\operatorname{ker}(M)}=\left\{a \in M \mid a^{n} \in \operatorname{ker}(M)\right\}$. Suppose $M$ is regular. For any $a \in \mathcal{R} \operatorname{ker}(M)$, there exists $e \in E(M)$ such that $M a M=M e M$. So

$$
e \in M e M=M a M \subseteq \mathcal{R} \operatorname{ker}(M)
$$

which implies $e \in \mathcal{R} \operatorname{ker}(M)$. Thus $e=e^{n} \in \operatorname{ker}(M)$. Therefore,

$$
M a M=M e M=\operatorname{ker}(M),
$$

and then $a \in \operatorname{ker}(M)$. This prove that $\mathcal{R} \operatorname{ker}(M)=\operatorname{ker}(M)$.

Suppose $\mathcal{R} \operatorname{ker}(M)=\operatorname{ker}(M)$. Let $G$ be the unit group of $M$. If $M$ is not regular, then $\overline{R(G)}$ is not completely regular following [22, Theorem 7.4]. Hence, from Theorem 4.1, there exist $a \in \overline{R(G)}$ and $f \in E\left(\operatorname{ker}(\overline{R(G)})\right.$, such that $a^{n}=f$ and $a \notin f \overline{R(G)} f$.

Let $x \in M$. Then $x \in \bar{B}$ for some Borel subgroup $B$ of $G$. Now $a \in \overline{R(G)} \subseteq \bar{B}$. From [22, Corollaries 1.16, 1.17, 3.20], we have that for all $b_{1}, b_{2} \in \bar{B}, b_{1} \mid b_{2}$ implies $b_{1}^{n} \mid b_{2}^{n}$. So $a \mid a x$ implies $a^{n} \mid(a x)^{n}$, i.e. $f \mid(a x)^{n}$. But $(a x)^{n} \mid f$ following $f \in E\left(\operatorname{ker}(\overline{R(G)})\right.$ and $E\left(\operatorname{ker}(\overline{R(G)}) \subseteq E(\operatorname{ker}(M))\right.$. So $f \mathscr{J}(a x)^{n}$. Thus $(a x)^{n} \in \operatorname{ker}(M), a x \in \sqrt{\operatorname{ker}(M)}$. This proves that $a M \subseteq \sqrt{\operatorname{ker}(M)}$. Thus by Lemma 3.3, $a \in \mathcal{R} \operatorname{ker}(M)=\operatorname{ker}(M)$. Since $a^{n}=f$, we have $f \in a M, f M \subseteq a M$. But by [9, Lemma 3(2)], $a M$ is a minimal right ideal of $M$ since $a \in \operatorname{ker}(M)$. So $f M=a M$, $a \in f M$. Similarly, $a \in M f$. Therefore, $a \in f M f$, and thus $a \in f \overline{R(G)} f$, a contradiction. So $\overline{R(G)}$ is completely regular, and hence $M$ is regular.

Finally, by Proposition 3.5, $\mathcal{R} \operatorname{ker}(M)$ is closed. Therefore, $\mathcal{R} \operatorname{ker}(M)$ is a linear algebraic semigroup which implies that $\mathcal{R} \operatorname{ker}(M)$ is an $s \pi r$-semigroup. Note that the kernel of $\mathcal{R} \operatorname{ker}(M)$ is just $\operatorname{ker}(M)$. Hence we have that $\mathcal{R} \operatorname{ker}(M)=\operatorname{ker}(M)$ if and only if $\mathcal{R} \operatorname{ker}(M)$ is completely simple.

\subsection{The solvability condition}

Theorem 4.3 Let $M$ be an irreducible linear monoid with unit group $G$ and $e \in$ $E(\operatorname{ker}(M))$. Then the following are equivalent:

(i) $G_{e}$ is solvable;

(ii) $|W(G)|=\left|W\left(H_{e}\right)\right|$;

(iii) $M$ is a semilattice of archimedean semigroups;

(iv) $\sqrt{\operatorname{ker}(M)}$ forms an ideal of $M$ (i.e., $\mathcal{R} \operatorname{ker}(M)=\sqrt{\operatorname{ker}(M)})$;

(v) $\mathcal{U}(M)$ is a relatively complement lattice.

Proof That (i) $\Longleftrightarrow$ (iii) $\Longleftrightarrow$ (v) follows from [20, Theorem 2.15]. Let $T$ be a maximal torus of $G$. By [22, Proposition 6.25] and [22, Theorem 6.16], we know 
that $|W(G)|=\omega(e) \mid W\left(C_{G}(e) \mid\right.$ where $\omega(e)=\left|J_{e} \cap E(\bar{T})\right|$, and $\left|W\left(C_{G}(e)\right)\right|=$ $\left|W\left(G_{e}\right)\right| \cdot\left|W\left(H_{e}\right)\right|$. Since $e \in E(\operatorname{ker}(M))$, we have $\omega(e)=1$, thus $|W(G)|=$ $\mid W\left(C_{G}(e)|=| W\left(G_{e}\right)|\cdot| W\left(H_{e}\right) \mid\right.$. So (i) $\Longleftrightarrow$ (ii).

By [22, Theorem 3.15], $M$ is isomorphic to a closed submonoid of $M_{n}(K)$ for some $n \in \mathbb{Z}^{+}$, thus

$$
\sqrt{\operatorname{ker}(M)}=\left\{a \in M \mid a^{n} \in \operatorname{ker}(M)\right\}
$$

If $M$ is a semilattice of archimedean semigroups, by [22, Theorem 1.15], for every $a, b \in M, a \mid b$ implies $a^{2} \mid b^{i}$ for some $i \in \mathbb{Z}^{+}$. Let $a \in \sqrt{\operatorname{ker}(M)}, b \in M$. since $a \mid a b$, we have $a^{n} \mid(a b)^{n}$. Moreover, $(a b)^{n} \mid a^{n}$ following $a^{n} \in \operatorname{ker}(M)$. So $a^{n} \mathscr{J}(a b)^{n}, a b \in \sqrt{\operatorname{ker}(M)}$. Similarly, $b a \in \sqrt{\operatorname{ker}(M)}$. Hence $M a M \in \sqrt{\operatorname{ker}(M)}$. This proves that $\sqrt{\operatorname{ker}(M)}$ is an ideal of $M$, that is $\mathcal{R} \operatorname{ker}(M)=\sqrt{\operatorname{ker}(M)}$. Thus (iii) $\Longrightarrow$ (iv).

Now we clam that (iv) $\Longrightarrow$ (iii). Suppose $\mathcal{R} \operatorname{ker}(M)=\sqrt{\operatorname{ker}(M)}$. Let $J \in \mathcal{U}(M)$ such that $J$ covers $\operatorname{ker}(M)$. By [19, Theorem 23, Remark 24], we just need to show that $\omega(J)=|J \cap E(\bar{T})|=1$. Suppose $\omega(J)>1$, then there exist $e, f \in J \cap E(\bar{T})$, such that $e \neq f$. By [22, corollary 6.8], there exists $x \in G$ such that $f=x_{e x}{ }^{-1}$. Since $e, f \in E(\bar{T}), e f=f e \in E(M)$. So $e f=f e \leq e, f$. But $e f \neq e$, ef $\neq f$. Thus ef $=f e \in E(\operatorname{ker}(M))$ as $J$ covers $\operatorname{ker}(M)$. Then exex ${ }^{-1}=$ xex $^{-1} e \in E(\operatorname{ker}(M))$. So exe $e x^{-1} e \in \operatorname{ker}(M)$. Hence

$$
(e x)(e x)=\left(\text { exe } x \in \operatorname{ker}(M),\left(x^{-1} e\right)\left(x^{-1} e\right)=x^{-1}\left(e x^{-1} e\right) \in \operatorname{ker}(M) .\right.
$$

Therefore, $e x, x^{-1} e \in \sqrt{\operatorname{ker}(M)}$. Since $\sqrt{\operatorname{ker}(M)}$ is an ideal of $M, e=(e x)\left(x^{-1} e\right) \in$ $\sqrt{\operatorname{ker}(M)}$, which implies that $e \in E(\operatorname{ker}(M))$, a contradiction. This shows that $\omega(J)=$ 1. Therefore, $M$ is a semilattice of archimedean semigroups.

Corollary 4.4 Let $M$ be an irreducible monoid with unit group $G$. Then $G$ is solvable if and only if $\sqrt{\operatorname{ker}(M)}$ forms an ideal of $M$ and a maximal subgroup of $\operatorname{ker}(M)$ is solvable.

Proof It is known that $G$ is solvable if and only if $|W(G)|=1$. Let $e \in E(\operatorname{ker}(M))$. From the proof of Theorem 4.3, we have that $|W(G)|=\left|W\left(G_{e}\right)\right| \cdot\left|W\left(H_{e}\right)\right|$, and $\left|W\left(G_{e}\right)\right|=1$ if and only if $\sqrt{\operatorname{ker}(M)}$ forms an ideal of $M$. So $G$ is solvable if and only if $\sqrt{\operatorname{ker}(M)}$ forms an ideal of $M$ and the subgroup $H_{e}$ is solvable.

\section{On completely regular $\mathcal{J}$-classes}

It is known that the kernel of a linear algebraic semigroup $S$ is a completely regular $\mathcal{J}$-class of $S$, and every completely regular $\mathcal{J}$-class of $S$ is completely simple (as a semigroup). In this section, we generalize the results about the kernel to completely regular $\mathcal{J}$-classes of linear algebraic semigroups.

The following construction plays an important role in this section. 
Proposition 5.1 Let $S$ be a linear algebraic semigroup. Let $J \in \mathcal{U}(S)$ be completely regular and let $S_{J}=\{a \in S \mid a J \subseteq J\}$. Then $S_{J}$ is a linear algebraic semigroup with kernel J. If $S$ is irreducible, $S_{J}$ is irreducible.

Proof By [22, Corollary 3.16], we can assume that $S$ is a closed subsemigroup of some $M_{n}(K)$. For any $a, b \in S_{J}, a b J \subseteq a J \subseteq J$, so $a b \in S_{J}$, which implies $S_{J}$ is a semigroup. Since $J$ is completely regular, $J^{2} \subseteq J$, thus $J \subseteq S_{J}$. Let $e \in E(J)$. Now we claim that $S_{J}=\left\{a \in S \mid \operatorname{det}_{e}(a) \neq 0\right\}$. Let $a \in S$ such that eae $\in J$. Then $e|e a| e a e \mid e$, thus $e a \in J$. Then for any $b \in J$, we have $e a b \in J$. So $a b|e a b| b \mid a b$, thus $a b \in J$ which implies $a J \subseteq J$. On the other hand, if $b \in S$ with $b J \subseteq J$, then ebe $\in J$ since $J^{2} \subseteq J$. Hence we get eae $\in J$ if and only if $a J \subseteq J$, i.e., $S_{J}=\left\{a \in S \mid \operatorname{det}_{e}(a) \neq 0\right\}$. Similarly, we have eae $\in J$ if and only if $J a \subseteq J$. But by [22, Remark 3.23], we obtain that $e a e \in J$ if and only if $\operatorname{det}_{e}(a) \neq 0$. So

$$
S_{J}=\{a \in S \mid J a \subseteq J\}=\left\{a \in S \mid \operatorname{det}_{e}(a) \neq 0\right\}
$$

Note that $J$ is an ideal of $S_{J}$ and $J$ is completely simple, thus $J$ is the kernel of $S$. Since $S_{J}=\left\{a \in S \mid \operatorname{det}_{e}(a) \neq 0\right\}$ which is open in $S, S_{J}$ is a linear algebraic semigroup with kernel $J$, and if $S$ is irreducible, $S_{J}$ is irreducible.

Throughout this section, we use the notation $S_{J}$ defined by

$$
S_{J}=\{a \in S \mid a J \subseteq J\}
$$

for a linear algebraic semigroup $S$ and a completely regular $\mathcal{J}$-class $J$ of $S$. Let $e \in E\left(S_{J}\right)$. By [22, Remark 1.3(iii)], the $\mathcal{H}$-class of $e$ in $S$ is equal to the $\mathcal{H}$-class of $e$ in $S_{J}$. Moreover, if $f \in E(S)$ with $e \mathcal{L} f$ in $S$, then $e f=e, f e=f$. Thus $f \in S_{J}$ and $e \mathcal{L} f$ in $S_{J}$. Therefore, we have that the two sets of idempotents of the $\mathcal{L}$-class of $e$ in $S$ and in $S_{J}$ coincide. Similarly, the two sets of idempotents of the $\mathcal{R}$-class of $e$ in $S$ and in $S_{J}$ coincide. Then applying the results of the structure of the kernel of $S$ described in [9] for $S_{J}$, it is easy to give the structure of completely regular $\mathcal{J}$-classes of $S$ by the following two corollaries directly.

Corollary 5.2 Let $S$ be a linear algebraic semigroup. Let $J \in \mathcal{U}(S)$ be completely regular. Then

$$
J=\bigcup_{h \in E(J)} H_{h}
$$

(ii) for any e, $f \in E(J), H_{e}$ is isomorphic to $H_{f}$ as an algebraic group under the morphism given by $x \mapsto g x f$ for some $g \in E(J)$.

Corollary 5.3 Let $S$ be a linear algebraic semigroup. Let $J \in \mathcal{U}(S)$ be completely regular, and $e \in E(J)$. Then

(i) $J=E\left(L_{e}\right) H_{e} E\left(R_{e}\right)$; 
(ii) under the Rees construction $J=E\left(L_{e}\right) \times H_{e} \times E\left(R_{e}\right)$,

$$
E(J)=\left\{\left(f,(g f)^{-1}, g\right) \mid f \in E\left(L_{e}\right), g \in E\left(R_{e}\right)\right\}
$$

which is isomorphic to $E\left(L_{e}\right) \times E\left(R_{e}\right)$ as an algebraic variety.

Theorem 5.4 Let $M$ be an irreducible linear algebraic monoid with unit group $G$, $J \in \mathcal{U}(M)$ and $e \in E(J)$. Then

$$
\operatorname{dim} J=2 \operatorname{dim} G+\operatorname{dim} H_{e}-\operatorname{dim} C_{G}^{r}(e)-\operatorname{dim} C_{G}^{l}(e) .
$$

In particular, if $J$ is completely regular,

$$
\operatorname{dim} J=\operatorname{dim} G-\operatorname{dim} G_{e} .
$$

Proof The algebraic group $G \times G$ acts on $M$ via left and right multiplication: $(g, h)$. $x=g x h^{-1}$. From [22, proposition 6.1], we know that the orbit of the element $e \in$ $E(M)$ under this action is just the $\mathcal{J}$-class of $e, J_{e}$. Consider $(G \times G)_{e}$, the isotropy subgroup of $e$ under the action of $G \times G$. Then

$$
\begin{aligned}
& (G \times G)_{e}=\left\{(x, y) \in G \times G \mid x e y^{-1}=e\right\} \\
& \quad=\{(x, y) \in G \times G \mid x e=e x e=e y e=e y\} .
\end{aligned}
$$

Hence $(G \times G)_{e}$ is a closed subgroup of $G \times G$, which contained in $C_{G}^{r}(e) \times C_{G}^{l}(e)$. By [22, Theorem 6.16] and its proof, we have

$$
\begin{aligned}
C_{G}^{r}(e) e & =e C_{G}^{l}(e)=e C_{G}(e)=H_{e}, \\
\operatorname{dim} C_{G}^{r}(e) & =\operatorname{dim} G^{r}(e)+\operatorname{dim} H_{e}, \operatorname{dim} C_{G}^{l}(e)=\operatorname{dim} G^{l}(e)+\operatorname{dim} H_{e},
\end{aligned}
$$

and $\operatorname{dim} C_{G}(e)=\operatorname{dim} G_{e}+\operatorname{dim} H_{e}$.

Let $\phi:(G \times G)_{e} \rightarrow H_{e} \times H_{e}$, defined by $(x, y) \mapsto(x e, e y)$. Obviously, $\phi$ is a homomorphism of algebraic groups. Then

$$
\phi\left((G \times G)_{e}\right)=\left\{(x e, e y) \in H_{e} \times H_{e} \mid x e=e y\right\}
$$

is isomorphic to $H_{e}$ as algebraic groups. Hence $\operatorname{dim} \phi\left((G \times G)_{e}\right)=\operatorname{dim} H_{e}$. Note that $\operatorname{ker}(\phi)=G^{r}(e) \times G^{l}(e)$. So

$\operatorname{dim}(G \times G)_{e}=\operatorname{dim} \phi\left((G \times G)_{e}\right)+\operatorname{dim} \operatorname{ker}(\phi)=\operatorname{dim} H_{e}+\operatorname{dim} G^{r}(e)+\operatorname{dim} G^{l}(e)$.

Since $J$ is the $G \times G$-orbit of $e$ in $M$ and $(G \times G)_{e}$ is the isotropy subgroup of $e$, we have $\operatorname{dim} J=\operatorname{dim}(G \times G)-\operatorname{dim}(G \times G)_{e}=2 \operatorname{dim} G-\operatorname{dim} H_{e}-\operatorname{dim} G^{r}(e)-\operatorname{dim} G^{l}(e)=$ $2 \operatorname{dim} G+\operatorname{dim} H_{e}-\operatorname{dim} C_{G}^{r}(e)-\operatorname{dim} C_{G}^{l}(e)$. 
If $J$ is completely regular and $e \in E(J)$, then by Theorem 2.2, $G=C_{G}^{l}(e) C_{G}^{r}(e)$. Hence $\operatorname{dim} G=\operatorname{dim} C_{G}^{l}(e)+\operatorname{dim} C_{G}^{r}(e)-\operatorname{dim} C_{G}(e)$. So

$$
\operatorname{dim} J=\operatorname{dim} G-\operatorname{dim} C_{G}(e)+\operatorname{dim} H_{e}=\operatorname{dim} G-\operatorname{dim} G_{e} .
$$

If $M$ is a linear algebraic monoid with unit group $G$, then the unit group of $M_{J}$ is exactly the unit group $G$ of $M$. Hence we can study the structure of the algebraic group $G$ in term of the data of completely regular $\mathcal{J}$-classes of $M$. By Theorem 2.2, $e \in E(\overline{R(G)})$ if only if $J_{e}$ is a completely regular $\mathcal{J}$-class of $M$. Then using the results about the kernel [8, Theorem 2.1], [10, Theorem 5.5], [11, Proposition 2.3, Theorem 2.4], we obtain the followings directly.

Corollary 5.5 Let $M$ be an irreducible linear algebraic monoid with unit group $G$, and let $e \in E(\overline{R(G)})$. Then

(i) $\operatorname{dim} R(G)=\operatorname{dim} E\left(J_{e}\right)+\operatorname{dim} R\left(G_{e}\right)+\operatorname{dim} R(e G e)$;

(ii) $\operatorname{dim} R_{u}(G)=\operatorname{dim} E\left(J_{e}\right)+\operatorname{dim} R_{u}\left(G_{e}\right)+\operatorname{dim} R_{u}(e G e)$.

(iii) $G$ is reductive if and only if $G_{e}$ and $J_{e}$ are both reductive groups.

Corollary 5.6 Let $M$ be an irreducible linear algebraic monoid with unit group $G$, and let $e \in E(\overline{R(G)})$. Let $P$ be a parabolic subgroup of $G$. Then

(i) $P=C_{P}(e) R(G)=C_{P}(e) R_{u}(G)$;

(ii) $P=C_{P}(e) \ltimes R_{u}(G)$ if and only if $G_{e}$ and $H_{e}$ are both reductive groups;

(iii) if $G_{e}$ is a reductive group and $J_{e}=E\left(J_{e}\right)$, then $P=P_{e} \ltimes R_{u}(G)$.

Now we want to generalize the results (Theorems 4.1, 4.2, 4.3) of the Schwarz radical to the case in terms of a completely regular $\mathcal{J}$-class $J$ in an irreducible linear algebraic monoid $M$. First, we give some properties of $M_{J}$.

Lemma 5.7 Let $M$ be an irreducible linear algebraic monoid. Let $J \in \mathcal{U}(M)$ be a completely regular $\mathcal{J}$-class and $e \in E(J)$. Then $a \mid e$ if and only if eae $\mid e$ for any $a \in M$.

Proof Let $G$ be the unit group of $M$, and $a \in M$. If eae $\mid e$, then $a|e a e| e$. So we only need to prove that if $a \mid e$, then eae $\mid e$. Suppose $a \mid e$. It follows from [22, Corollary 6.13] that $a \in G M_{e} G$, and thus there exist $x, y \in G$ and $b \in M_{e}$ such that $a=x b y$. Thus eae $=$ exbye. And exbe $=$ exe He following Theorem 2.2. So exb $\mid e$ which implies $e x b \in J$. Clearly, ye $\in J$. Since $J$ is completely regular, $J^{2}=J$. Hence $e a e=$ exbye $\in J$ and thus eae $\mid e$.

Proposition 5.8 Let $M$ be an irreducible linear algebraic monoid with unit group $G$, and let $J \in \mathcal{U}(M)$ be a completely regular $\mathcal{J}$-class, $e \in E(J)$. Let $S=M \backslash G=$ $\cup_{i \in I} S_{i}$, where $S_{i}$ are the irreducible components of $S$. Then

$$
M_{J}=\{a|a \in M, a| e\}=G M_{e} G=M \backslash\left(\cup_{i \in \Delta} S_{i}\right),
$$

where $\Delta=\left\{i \in I \mid\right.$ for all $\left.a \in S_{i}, a \nmid e\right\}$. 
Proof Given an element $a \in M$, by Lemma 5.7, we have $a \mid e$ if and only if eae $\mid e$. By the proof of Proposition 5.1, $M_{J}=\{a \mid a \in M$, eae $\mid e\}$. Hence

$$
M_{J}=\{a|a \in M, a| e\}=G M_{e} G
$$

following [22, Corollary 6.13]. In particular,

$$
a \nmid e \Longleftrightarrow e a e \nmid e \Longleftrightarrow e a e \notin H_{e} \Longleftrightarrow \operatorname{det}_{e}(a)=0 \text {. }
$$

Let $I(e)=\{a \mid a \in M, a \nmid e\}$. Then $I(e)=\left\{a \mid a \in M, \operatorname{det}_{e}(a)=0\right\}$ and thus $M \backslash M_{J}=I(e)$.

If $M=G$, then $S=\varnothing$ and $M_{J}=G$. And if $J=\operatorname{ker}(M)$, then $M_{J}=M$. Suppose $M \neq G$ and $J \neq \operatorname{ker}(M)$. Now we claim that $I(e)=\cup_{i \in \Delta} S_{i}$, where $\Delta=\left\{i \in I \mid\right.$ for all $\left.a \in S_{i}, a \nmid e\right\}$. By [22, Theorem 3.15], we can assume that $M$ is a closed submonoid of some $M_{n}(K)$. Consider $\phi: M \rightarrow K$ given by $\phi(a)=\operatorname{det}_{e}(a)=\operatorname{det}(e a e+1-e)$. Thus $I(e)=\phi^{-1}(0)$. By [22, Theorem 2.21], the dimension of every irreducible component of $I(e)$ is $p-1$. Since $J$ is completely regular, $\omega(e)=1$. From Theorem 2.2, we have $e G e$ is the $\mathcal{H}$-class of $e$. Thus if $a \in G$, then eae $\in H_{e}$, which implies $a \notin I(e)$. So $I(e) \subseteq M \backslash G=S$. Moreover, by [22, Proposition 6.2], the dimension of every irreducible component of $S$ is $p-1$. Hence every irreducible component of $I(e)$ is also the irreducible component of $S$. Let $S_{0}$ be an irreducible component of $S$ which satisfy that for any element $a \in S_{0}, a \nmid e$. Then $S_{0} \subseteq I(e)$. Hence $I(e)=\cup_{i \in \Delta} S_{i}$. Therefore, $M_{J}=M \backslash I(e)=M \backslash\left(\cup_{i \in \Delta} S_{i}\right)$.

Corollary 5.9 Let $M$ be an irreducible submonoid of $M_{n}(K)$ with unit group $G$, and let $J \in \mathcal{U}(M)$ be a completely regular $\mathcal{J}$-class. Then

$$
\left\{a \in M \mid a^{n} \in J\right\}=\left\{a \in M_{J} \mid a^{n} \in J\right\} .
$$

Proof If $a \in M$ with $a^{n} \in J$. Let $e \in E(J)$. Then $a\left|a^{n}\right| e$. So $a \notin I(e)$. By Proposition 5.8, $a \in M_{J}$. Hence $\left\{a \in M \mid a^{n} \in J\right\}=\left\{a \in M_{J} \mid a^{n} \in J\right\}$.

For an irreducible linear algebraic monoid $M$ with unit group $G$. Let $J \in \mathcal{U}(M)$ be a completely regular $\mathcal{J}$-class, we denote

$$
\sqrt{J}=\left\{a \in M \mid a^{i} \in J \text { for some } i \in \mathbb{Z}^{+}\right\}
$$

and $\mathcal{R}(J)=\{a \in M \mid G a G \subseteq \sqrt{J}\}$. By Proposition 3.4 and Corollary 5.9, it is easy to see that the $S c h w a r z$ radical $\mathcal{R} \operatorname{ker}\left(M_{J}\right)$ of $M_{J}$ is equal to $\mathcal{R}(J)$. Moreover, $J, \sqrt{J}$ and $\mathcal{R}(J)$ are both affine variety. This is because $M_{J}$ is an affine variety and $J, \sqrt{J}$, $\mathcal{R}(J)$ are both closed in $M_{J}$.

For an irreducible linear algebraic monoid $M$ with unit group $G$, there is a natural algebraic group $G \times G$ acts on $M$ via left and right multiplication:

$$
(g, h) \cdot x=g x h^{-1} \text { for } g, h \in G \text { and } a \in M \text {. }
$$


From [22, Proposition 6.1], we know that the orbit of an element $a \in M$ under this action is just the $\mathcal{J}$-class of $a, J_{a}$. Now we can give a generalization of the results in Sect. 4 in the language of linear algebraic group actions.

Corollary 5.10 Let $M$ be an irreducible linear algebraic monoid with unit group $G$, and let $J \in \mathcal{U}(M)$ be a completely regular $\mathcal{J}$-class, $e \in E(J)$. Then

(i) $G_{e}$ is reductive if and only if $J$ is the unique $G \times G$-stable affine subvariety of $M$ contained in $\sqrt{J}$ (i.e., $\mathcal{R}(J)=J$ );

(ii) $G_{e}$ is solvable if and only if $\sqrt{J}$ is a $G \times G$-stable affine subvariety of $M$ (i.e., $\mathcal{R}(J)=\sqrt{J})$

Proof Let $M_{J}=\{a \in M \mid a J \subseteq J\}$. By Proposition 5.1, $M_{J}$ is an irreducible linear algebraic monoid with kernel $J$. Obviously, the unit group of $M_{J}$ is $G$. Moreover, as we see above, the radical of the kernel of $M_{J}$ is equal to $\sqrt{J}$, and $\mathcal{R} \operatorname{ker}\left(M_{J}\right)=$ $\mathcal{R}_{M_{J}}(J)=\mathcal{R}(J)$. By [22, Theorem 7.4], $M_{J}$ is regular if and only if $G_{e}$ is reductive since $e \in E(J)$. Apply Theorems 4.2 and 4.3 for $M_{J}$, we have $G_{e}$ is reductive if and only if $\mathcal{R}(J)=J$, and $G_{e}$ is solvable if and only if $\mathcal{R}(J)=\sqrt{J}$. Since $\mathcal{R}(J)=\{a \in M \mid G a G \subseteq \sqrt{J}\}$ and $J$ is the $G \times G$-orbit of $e$ in $M$, we have that that $\mathcal{R}(J)=J$ is equivalent to that $J$ is the unique $G \times G$-stable affine subvariety of $M$ contained in $\sqrt{J}$, and $\mathcal{R}(J)=\sqrt{J}$ is equivalent to that $\sqrt{J}$ is a $G \times G$-stable affine subvariety of $M$.

As an immediate consequence of Corollary 5.5(iii), 5.10 and [22, Proposition 6.25] we have,

Corollary 5.11 Let $M$ be an irreducible linear algebraic monoid with unit group $G$, let $J \in \mathcal{U}(M)$ be a completely regular $\mathcal{J}$-class. Then

(i) $G$ is reductive if and only if $J$ is both a reductive group and the unique $G \times G$ stable affine subvariety of $M$ contained in $\sqrt{J}$;

(ii) $G$ is solvable if and only if $\sqrt{J}$ is a $G \times G$-stable affine variety and a maximal subgroup of $\mathrm{J}$ is solvable.

Remark 5.12 Let $M$ be an irreducible solvable linear algebraic monoid. Since $M$ is an $s \pi r$-semigroup, it is easy to verify that

$$
M=\bigcup_{J \in \mathcal{U}(M)} \sqrt{J}
$$

which is a semilattice of $\sqrt{J}(J \in \mathcal{U}(M))$, and every $\sqrt{J}$ is an archimedean semigroup and a nil extension of the completely simple semigroup $J$.

Acknowledgements I would like to thank Professors Wenxue Huang and Mohan Putcha for encouragement and helpful discussion during preparing for this article. This work is partially supported by NSFC Grant (11171202).

Open Access This article is distributed under the terms of the Creative Commons Attribution 4.0 International License (http://creativecommons.org/licenses/by/4.0/), which permits unrestricted use, distribution, and reproduction in any medium, provided you give appropriate credit to the original author(s) and the source, provide a link to the Creative Commons license, and indicate if changes were made. 


\section{References}

1. Bogdanović, S., Ćirić, M.: Semigroups in which the radical of every ideal is a subsemigroup. Zbornik radova Fil. fak. Niš 6, 129-135 (1992)

2. Borel, A.: Linear Algebraic Groups, 2nd edn. Springer, Berlin (1991)

3. Bosak, J.: On radicals of semigroups. Mat. casop. 18, 204-212 (1968)

4. Brion, M.: Local structure of algebraic monoids. Moscow Math. J. 8, 647-666 (2008)

5. Ćirić, M., Bogdanović, S.: Decompositions of semigroups induced by identities. Semigr. Forum 46, 329-346 (1993)

6. Clifford, A.H.: Semigrouips without nilpotent ideals. Am. J. Math. Soc. 71, 834-844 (1949)

7. Demazure, M., Gabriel, P.: Introduction to Algebraic Geometry and Algebraic Groups, 1st edn. NorthHolland, Amsterdam (1980)

8. Huang, W.: Reductive and semisimple algebraic monoids. Forum Math. 13, 495-504 (2001)

9. Huang, W.: The kernel of a linear algebraic semigroup. Forum Math. 17, 851-869 (2005)

10. Huang, W.: Kernels, regularity and unipotent radicals in linear algebraic monoids. Forum Math. 23, 803-834 (2011)

11. Huang, W.: Parabolic subgroups and algebraic monoids. J. Algebra 336, 227-235 (2011)

12. Huang, W.: The structure of affine algebraic monoids in terms of kernel data. Fields Lust. Commun. 71, 119-140 (2014)

13. Humphreys, J.E.: Linear Algebraic Groups, Corrected, 3rd edn. Springer, Berlin (1981)

14. Kmet, F.: On radicals in semigroups. Math. Slovaca 32, 204-212 (1968)

15. Kmet, F.: Radicals and their left ideal analogues in a semigroup. Math. Slovaca 38, 139-145 (1988)

16. Koch, R.J.: Remarks on primitive idempotents in compact semigroups with zero. Proc. Am. Math. Soc. 5, 828-833 (1954)

17. Luh, J.: On the concepts of radical of semigroup having kernel. Port. Math. 19, 189-198 (1960)

18. Putcha, M.S.: On algebraic semigroups, II. Trans. Am. Math. Soc. 259, 471-491 (1980)

19. Putcha, M.S.: The $\mathscr{J}$-class structure of connected algebaraic monoids. J. Algebraic 73, 601-612 (1981)

20. Putcha, M.S.: Connected algebraic monoids. Trans. Am. Math. Soc. 272, 693-709 (1982)

21. Putcha, M.S.: Reductive groups and regular semigroups. Semigr. Forum 30, 253-261 (1984)

22. Putcha, M.S.: Linear Algebraic Monoids. London Mathematical Society Lecture Note Series 133. Cambridge University Press, Cambridge (1988)

23. Renner, L.E.: Linear algebraic monoids. In: Encyclopaedia in Mathematical Sciences, vol. 99. Invariant Theory and Algebraic Transformation Groups, V. Springer, Berlin (2005)

24. Schwarz, S.: Zur theorie der halbgruppen, vol. 6. Sbornk Prc Prrodovedeckej Fakulty Slovenskej Univerzity v Bratislave (1943)

25. Schwarz, S.: On semigroups having a kernel. Czechoslovak Math. J. 1, 76 (1951)

26. Springer, T.A.: Linear Algebraic Groups, 2nd edn. Birkhäuser, Boston (1998) 